\section{Immune to weight loss} L eptin always seemed a bit too good to be true. Why would our chronically undernourished ancestors have evolved a hormone for weight loss? Now, Ke Chen, Allan Zhao (University of Pittsburgh, PA), and colleagues explain why leptin doesn't work as the weight loss wonder drug that initially seemed a possibility.

Leptin was discovered as a hormone produced by fat cells. When reinstated in leptin-deficient mice and humans it drastically reduced their obesity. But individuals who were obese for other reasons had paradoxically high leptin levels, and adding more leptin did not induce significant weight loss.

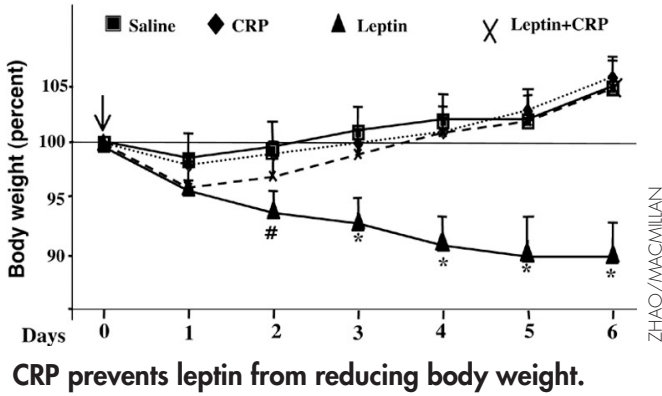

The Pittsburgh group looked to leptin-binding proteins for an answer. The first that they found was $\mathrm{C}$-reactive protein (CRP), which is famous as a marker for immune reactions but whose exact function remains mysterious. CRP bound to leptin, and inhibited leptin signaling in tissue culture. In mice lacking their own leptin, CRP reduced or eliminated the effects of added leptin on food intake, body weight, blood glucose, and lipid metabolism.

The CRP connection is not completely without precedent, as others have noted inflammation (and elevated CRP) as a complication of obesity. The adipogenic and inflammatory systems also share many of the same mediators, so there is plenty of potential for crosstalk. It is not clear whether this is an accident of evolution or has a particular function.

Zhao's one clue is that low levels of leptin turn on CRP expression. He suspects CRP helped our ancestors to accumulate some fat in the few periods of plenty interspersed amongst the more common lean times. "If leptin worked unimpeded you would have a very difficult time accumulating fat," he says.

Now that energy rich food is always available, however, the system has become pathological. Zhao's next step is to find out whether those who are morbidly obese have a different set point for CRP expression or accumulation. JCB

Reference: Chen, K., et al. 2006. Nat. Med. 12:425-432.

\section{Mixed-up DNA}

nterphase human chromosomes show significant intermingling, say Miguel Branco and Ana Pombo (MRC Clinical Sciences Centre, London, UK). The idea is a counter to the most extreme view of chromosome territories.

Territories were so named based on microscopists' early observations: that individual chromosomes did not spread out over an entire nucleus. DNA labeling also indicated that nuclei had gaps between DNA, although it was not clear if these were between DNA of the same or different chromosomes. Still, this was enough to spark talk of an interchromatin domain separating territories. "In this model, it will always be right because two pieces of chromosome will never be in exactly the same place," says Pombo. "The field couldn't move forward."

She and Branco responded by labeling two different chromosomes in ultrathin slices and following up with EM for even higher resolution. Based on extrapolation, an average of $46 \%$ of each chromosome territory intermingles with other chromosomes. Furthermore, extents of intermingling for different chromosomes correlated with translocation frequencies.

The nucleus is not a complete tangled mess, however. "Chromosomes as polymers will tend to expand," says Pombo. "But what stops them is interaction with each other and with the nuclear membrane." She is now seeing if transcription factories help keep particular chromosomes fixed to themselves or each other. JCB

Reference: Branco, M.R., and A. Pombo. 2006. PLoS. 4:e138.

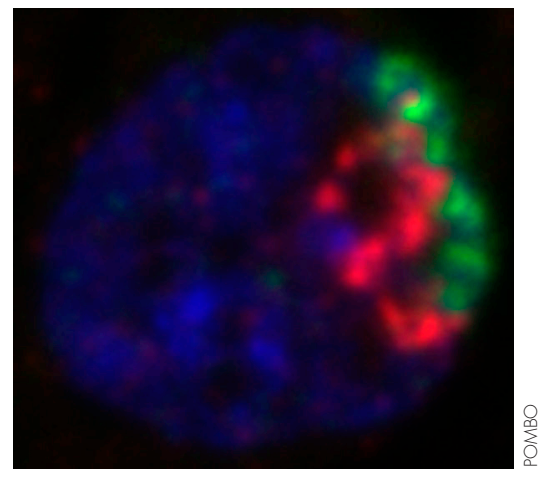

Two chomosomes (red and green) mingle.

\title{
Stressful betrayal
}

$\mathrm{D}$ uring heat shock recovery, a helper becomes a destroyer, say ShuBing Qian, Cam Patterson, and colleagues (University of North Carolina, Chapel Hill, NC).

The traitor protein is called CHIP. After the group discovered CHIP, their interest was piqued when they realized it was both an Hsp70-binding protein and a ubiquitin ligase. The finding suggested that "folding versus degradation is a regulated decision, not a stochastic decision, and CHIP ties together these two processes," says Patterson.

They now find that CHIP helps to mark unfolded proteins that are bound to Hsp70 by adding the ubiquitin destruction signal. But once those marked proteins are destroyed, the Hsp70 itself becomes CHIP's next target. The effect could

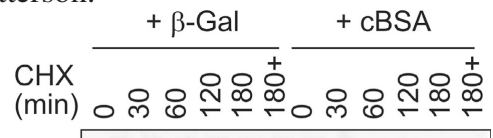
Hsp70 - -

Hsp70 gets destroyed (left) unless unfolded protein (cBSA; right) is around.

be reconstituted in vitro with only unfolded protein, Hsp70, and CHIP.

The resultant return to baseline Hsp70 levels is important to restore cellular normality. Hsp70 helps halt the cell cycle and alter signaling pathways so that cells can recover from stress. Once unfolded proteins are cleared, says Patterson, "the cell has to get out of this suspended animation phase." JCB

Reference: Qian, S.-B., et al. 2006. Nature. 440:551-555. 